\title{
ON FRENET EQUATIONS FOR CURVES OF CLASS $C^{\infty}$
}

\author{
KATSUMI NOMIZU
}

(Received March 28, 1958, Revised September 10,1958)

The purpose of the present note is to clarify certain ambiguities which appear in the usual treatment of the fundamental theorem of curves in 3 dimensional Euclidean space. The theorem in its classical form may ke stated in the following two parts.

THEOREM I. Given a curve in Euclidean space which is defined by $x^{i}=$ $x^{i}(s)^{1)}$, where $x^{i}$ are functions of class $C^{3}$ on an interval $L=[0, \chi]$ and $s$ is the arc-length measured from the initial point $x^{i}(0)$, there exists a family of ortho-normal vectors $e_{i}(s)$ which satisfy the Frenet equations

$$
\begin{aligned}
& d e_{1} / d s=k e_{2} \\
& d e_{2} / d s=-k e_{1}+w e_{3} \\
& d e_{3} / d s=-w e_{2}
\end{aligned}
$$

where $e_{1}$, and $e_{2} e_{3}$ are the tangent, principal normal and binormal unit vectors respectively and $k, w$ are the curvature and torsion respectively. It is assumed, however, that the curvature $k(s)$ (defined to be the length of the derivative $d e_{1} / d s$ of the unit tangent vector $e_{1}$ ) does not vanish anywhere on $L$.

THEOREM II. Given a function $k(s)$ of class $C^{1}$ and a continuous function w(s) on an interval $L$, there exists a curve of class $C^{3}$ which admits a family of ortho-normal vectors $e_{1}, e_{2}$ and $e_{3}$ satisfying the equations (1) with given functions $k$ and $w$, where $e_{1}$ is the unit tangent vector. Such a curve is uniquely determined by $k$ and w within to a motion of the space.

The question of weakening differentiability requirements of the curve in Theorem I and of the functions $k$ and $w$ in Theorem II has been studied by P. Hartman and A. Wintner ${ }^{2)}$. What we wish to take up here is the assumption usually made for Theorem I that the curvature $k$ does not vanish anywhere on the whole interval $L$; the other extreme case is that of a line segment for which $k$ is constantly zero. This assumption of non-vanishing curvature is not satisfactory in view of Theorem II in which the given func-

1) Throughout the paper, the suffix $i$ runs from 1 to 3 .

2) P. Hartman and A. Wintner, On the fundamental equations of differential geometry, Amer. J. Math, 72 (1950); A. Wintner, On the infinitesimal geometry of curves, Amer. J. Math. 75 (1953). 
tions $k$ and $w$ are quite arbitrary. What is then a necessary and sufficient condition for a given curve to admit a family of ortho-normal vectors $e_{1}$ (=unit tangent vector), $e_{2}$ and $e_{3}$ which satisfy (1) with suitably chosen functions $k$ and $w$ ? A partial answer to this problem and its applications to the theory of surfaces have been given by A. Wintner ${ }^{3}$.

In this note, we assume that the functions $x^{i}(s)$ defining the curves are of class $C^{\infty}$ and analyze conditions for establishing the Frenet equations without requiring that the function $k(s)$ is non-negative.

1. Regular, normal and Frenet curves. Let $x^{i}$ be rectangular coordinates in Euclidean space $R$. By a parametrized curve of class $C^{\infty}$, we mean a mapping $f$ of a certain interval $L=[a, b]$ into $R$ defined by $x^{i}=x^{i}(t)$, $a \leqq t \leqq b$, where $x^{i}(t)$ are functions of class $C^{\infty}$ on $L^{4}$. Two parametrized curves of class $C^{\infty} f: x^{i}=x^{i}(t), a \leqq t \leqq b$, and $\bar{f}: x^{i}=\bar{x}^{i}(\bar{t}), \bar{a} \quad \bar{t} \quad \bar{b}$, are said to te equivalent if there exists a diffeomorphism $\bar{t}=\phi_{(}(t)$ of class $C^{\infty}$ of $[a, b]$ onto $[\bar{a}, \bar{b}]$ such that $\phi^{\prime}(a)=\bar{a}, \phi^{\prime}(b)=\bar{b}$ and $x^{i}(t)=\overline{x^{i}}(\phi(t))$ for every $t$ in $[a, b]$. Each equivalence class of parametrized curves of class $C^{\infty}$ will be called an oriented curve of class $C^{\infty}$ or simply a curve.

Let $C$ be a curve defined by $x^{i}=x^{i}(t), a \leqq t \leqq b$. If $\sum_{i=1}^{3}\left(d x^{i} / d t\right)^{2} \neq 0$ for every $t$, then $C$ is called a regular curve. Of course, this notion is independent of the choice of a parametrized curve $x^{i}=x^{i}(t)$ which represents the curve $C$. If $C$ is a regular curve, then we may introduce the arc-length $s=\int_{a}^{t} \sqrt{\sum_{i=1}^{3}\left(d x^{i} / d t\right)^{2}} d t$ by means of which we can represent $C$ in the form $x^{i}=x^{i}(s), 0 \leqq s \leqq \chi$, where $\chi$ is the total length of $C$. From now on, we consider only regular curves which are represented in this form. The vector $e_{1}(s)=\left(d x^{i} / d s\right)$ of length 1 is called the unit tangent vector at the point $x^{i}(s)$.

A regular curve will here be called nor mal if it satisfies the following condition: for every $s_{0} \in L$, there exists an integer $m=m\left(s_{0}\right)$ such that the $m$-th derivative $\left(d^{m} e_{1} / d s^{m}\right)\left(s_{0}\right)$ at $s_{0}$ is not zero. It is clear that an analytic curve is normal unless it is a line segment.

A regular curve $x^{i}=x^{i}(s)$ will be called a Frenet curve if there exists a family of vectors $e_{2}(s)$ and $e_{3}(s), s \in L$, such that ${ }^{5)}$

1) for each $s, e_{1}(s), e_{2}(s)$ and $e_{3}(s)$ are ortho-normal (that is, they are mutually orthogonal unit vectors) and the matrix $\left\|e_{1}(s) e_{2}(s) e_{3}(s)\right\|$ is of de-

3) A. Wintner, On Frenet's equations, Amer. J. Math. 78(1956).

4) By a $C^{\infty}$-function on a closed interval $L$, we mean a function which may be extended to a $C^{\infty}$-function on an open interval containing $L$.

5) In accordance with 4), we assume that $e_{i}(s)$ can be extended to an open interval containing $L$ so as to satisfy the following conditions there. 
terminant 1 ;

2) $e_{2}(s)$ and $e_{3}(s)$ are of class $C^{\infty}$ with respect to $s$;

3 ) these vectors satisfy the Frenet equations (1) with suitably chosen functions $k$ and $w$.

Such a family $\left(e_{1}, e_{2}, e_{3}\right)$ is called a Frenet family of moving frames on the Frenet curve $C$. The family of unit tangent vectors $e_{1}(s)$ is of course of class $C^{\infty}$. The functions $k$ and $w$, which are equal to the inner product $\left(d e_{1} / d s, e_{2}\right)$ and $-\left(d e_{3} / d s, e_{2}\right)$ respectively, are automatically of class $C^{\infty}$. The Frenet family of moving frames of a Frenet curve $C$ may not be unique as the case of a line segment clearly illustrates (here $k$ is constantly zero, but we may choose $e_{2}$ and $e_{3}$ so that $w$ may be an arbitrary pressigned function of class $\left.C^{\infty}\right)$. The absolute value of the function $k(s)$ is uniquely determined as the length of $d e_{1} / d s$; the function $w(s)$ is unique if we choose one fixed family of moving frames.

We denote by $e_{1}{ }^{(m)}$ the $m$-th derivative $d^{m} e_{1} / d s^{m}$ of $e_{1}(s)$. Applying the Leibniz formula to the first equation of (1), we see that if $e_{1}^{\prime}=\ldots=e_{1}{ }^{(m)}=0$ and $e_{1}{ }^{(m+1)} \neq 0$ at a point $s=s_{0}$, then $k\left(s_{0}\right)=\ldots=k^{(m-1)}\left(s_{0}\right)=0$ and $k^{(m)}\left(s_{0}\right) \neq 0$.

2. Main results. Our main results are the following.

THEOREM. A normal curve of class $C^{\infty}$ is a Frenet curve. The function $k(s)$ which appears in the Frenet equations of $C$ is unique up to a sign and the function $w(s)$ is uniquely determined.

COROLlARY 1. Let $C$ be a normal curve of class $C^{\infty}: x^{i}=x^{i}(s), s \in L$ $=[0, \chi]$. For each $s_{0} \in L$, denote by $m\left(s_{0}\right)$ the first integer $m$ such that $e_{1}^{(m)}\left(s_{0}\right)$ is not zero. If $m\left(s_{0}\right)$ is odd, the function $k(s)$ does not change its sign at $s=s_{0}$, while if $m\left(s_{0}\right)$ is even, $k(s)$ changes its sign at $s=s_{0}$.

The geometric meaning of Corollary 1 will be explained later. We define $k(s)$ or $-k(s)$ as the curvature of $C$ and the function $w^{\prime}(s)$ as the torsion of C.

COROLLARY 2. An analytic curve is always a Frenet curve.

The proof of the theorem is preceded by the following lemmas.

LEMMA 1. Let $f(s)$ be a $C^{\infty}$-function of $s$ defined on a certain interval L. If a sequence of points $s_{n} \in L$ with $f\left(s_{n}\right)=0$ converges to a point $s_{0} \in L$, then the derivatives of all orders $f^{(m)}\left(s_{0}\right)$ are zero.

PROOF. That $f\left(s_{0}\right)=0$ is obvious. In order to prove that $f^{\prime}\left(s_{0}\right)=0$, we may assume that $s_{n}$ converges to $s_{0}$ monotonely, say, $s_{1}>s_{2}>\ldots>s_{n} \ldots$ By Rolle's theorem, there exists a sequence of points $t_{n}$ such that $f^{\prime}\left(t_{n}\right)=0$ and $s_{n}>t_{n}>s_{n+1}$ for all $n$. Then $t_{n}$ converge to $s_{0}$ and hence $f^{\prime}\left(s_{0}\right)=0$. By 
repeated application of this argument,we have $f^{(m)}\left(s_{0}\right)=0$ for all $m$.

Lemma 1 is valid also for a vector-valued function $e_{1}(s)=\left(d x^{i} / d s\right)$ of a regular curve of class $C^{\infty}$. Thus we get

LEMMA 2. Let $C$ be a normal curve of class $C^{\infty}$ represented by $x^{i}=x^{i}(s)$, $s \in L$. Then the set $N$ of points $s \in L$ where $e_{1}^{\prime}(s)=0$ consists of isolated points and hence is finite ( $N$ may be empty).

LEMMA 3. (Taylor theorem for $C^{\infty}$-functions). Let $f(s)$ be a function of class $C^{\infty}$ defined in a neighborhood $U$ of $s_{0}$. For each integer $m \geqq 1$, there exists a $C^{\infty}$-function $g(s)$ on $U$ such that

$$
f(s)=f\left(s_{0}\right)+\left(s-s_{0}\right) f^{\prime}\left(s_{0}\right)+\ldots+\frac{\left(s-s_{0}\right)^{m}}{m !} f^{(m)}\left(s_{0}\right)+\left(s-s_{0}\right)^{m+1} g(s)
$$

for all $s$ in $U$.

PROOF. This follows from the following well known fact. If $f(s)$ is a $C^{\infty}$-functon such that $f\left(s_{0}\right)=0$, then there exists a $C^{\infty}$-function $g(s)$ such that $f(s)=\left(s-s_{0}\right) g(s)$.

With these preparations we are now in a position to prove our theorem.

By. Lemma 2. $e_{1}^{\prime}(s)$ vanishes on a finite set $N=\left\{s_{1}, s_{2}, \ldots, s_{p}\right\}$ of $L$. We assume that $0<s_{1}<s_{2}<\ldots<s_{p}<\chi$ (the case where $s_{1}=0$ or $s_{p}=\chi$ can ke handled in a similar way). Let us define the vectors $e_{2}(s)$ and the function $k(s)$ on $L$ in the following manner. For $0 \leqq s<s_{1}$, we define

$$
e_{2}(s)=e_{1}^{\prime}(s) /\left\|e_{1}^{\prime}(s)\right\| \quad \text { and } \quad k(s)=\left\|e_{1}^{\prime}(s)\right\|,
$$

where $\|\quad\|$ denotes the length.

In the case where $m=m\left(s_{1}\right)$ is odd, we define

and for $s_{1}<s<s_{2}$

$$
e_{2}\left(s_{1}\right)=e_{1}{ }^{(m)}\left(s_{1}\right) /\left\|e_{1}{ }^{(m)}\left(s_{1}\right)\right\| \quad \text { and } \quad k\left(s_{1}\right)=0 \text {, }
$$

$$
e_{2}(s)=e_{1}^{\prime}(s) /\left\|e_{1}^{\prime}(s)\right\| \quad \text { and } \quad k(s)=\left\|e_{1}^{\prime}(s)\right\| .
$$

In the case where $m=m\left(s_{1}\right)$ is even, we define

$$
e_{2}\left(s_{1}\right)=-e_{1}{ }^{(m)}\left(s_{1}\right) /\left\|e_{1}{ }^{(m)}\left(s_{1}\right)\right\| \quad \text { and } \quad k\left(s_{1}\right)=0,
$$

and for $s_{1}<s<s_{2}$

$$
e_{2}(s)=-e_{1}^{\prime}(s) /\left\|e_{1}^{\prime}(s)\right\| \quad \text { and } \quad k(s)=-\left\|e_{1}^{\prime}(s)\right\| .
$$

We continue this process of defining $e_{2}(s)$ and $k(s)$ successively on each subinterval $\left(s_{i}, s_{i+1}\right)$ and at the points $s_{i}$ in such a way that the function $k(s)$ does not change its sign at $s=s_{i}$ if $m\left(s_{i}\right)$ is odd ard changes its sign at $s=s_{i}$ if $m\left(s_{i}\right)$ is even. After defining $e_{2}(s)$ on the whole interval $L$, we set $e_{3}(s)=e_{1}(s) \times e_{2}(s)$ for every $s$. We shall prove that $e_{1}(s), e_{2}(s)$ and $e_{3}(s)$ form actually a Frenet family of moving frames of $C$.

First observe that $\left(e_{1}, e_{1}\right)=1$ implies $\left(e_{1}, e_{1}^{\prime}\right)=0$, which shows that $e_{1}(s)$ 
and $e_{2}(s)$ are orthogonal to each other for $s \in L-N$. If $s_{i} \in N$, we have $e_{1}^{\prime}=\ldots=e_{1}^{m\left(s_{i}\right)-1}=0$ at $s=s_{i}$. Further differentiation of $\left(e_{1}, e_{1}\right)=1$ gives $\left(e_{1}, e_{1}^{m\left(s_{i}\right)}\right)=0$ at $s=s_{i}$. Hence $e_{1}\left(s_{i}\right)$ and $e_{2}\left(s_{i}\right)$ are orthogonal to each other. This shows that $e_{1}(s)$ and $e_{2}(s)$ are unit vectors orthogonal to each other for every $s$ so that $e_{1}(s), e_{2}(s)$ and $e_{3}(s)$ satisfy condition 1$)$ of the Frenet moving frames. If we know that they are difterentiable, then the classical argument establishes the Frenet equations by suitably defining $w(s)$.

It remains therefore to show that $e_{2}(s)$ is of class $C^{\infty}$. Since $e_{1}(s)$ is of class $C^{\infty}$, that will imply that $e_{3}(s)$ is of class $C^{\infty}$. On each subinterval $\left(s_{i}, s_{i+1}\right), e_{2}(s)$ is clearly of class $C^{\infty}$. It suffices therefore to show that $e_{2}(s)$ is of class $C^{\infty}$ in a neighborhood of each point $s_{i}$ of $N$.

Let $s_{i} \in N$ and let $U=\left(s_{i-1}, s_{i+1}\right)$. We introduce the following notations : $m=m\left(s_{i}\right), \quad h=s-s_{i}(s \in U), \quad e_{1}^{\prime}(s)=\left(\xi^{i}(h)\right), \quad e_{1}{ }^{(m)}\left(s_{i}\right)=\left((m-1) ! a^{i}\right)$. By Lemma 3 , there exist $C^{\infty}$-functions $\eta^{i}(h)$ such that

$$
\xi^{i}(h)=h^{m-1} a^{i}+h^{m} \eta^{i}(h)
$$

First consider the case where $m$ is odd. We shall assume that $e_{2}(s)=e_{1}^{\prime}(s) /\left\|e_{1}^{\prime}(s)\right\|$ for $s_{i-1}<s<s_{i}$, since the case of the opposite sign may be treated similarly. Then we have

$$
i \text {-th component of } e_{2}(s)=\frac{h^{m-1} a^{i}+h^{m} \eta^{i}(h)}{\left(\sum_{i=1}^{3}\left(h^{m-1} a^{i}+h^{m} \eta^{i}(h)\right)^{2}\right)^{1 / 2}}
$$

for $s_{i-1}<s<s_{i}$. As $m$ is odd, we have

$$
i \text {-th component of } e_{2}(s)=\frac{a^{i}+h \eta^{i}(h)}{\left(\sum_{i=1}^{3}\left(h^{m-1} a^{i}+h^{m} \eta^{i}(h)\right)^{2}\right)^{1 / 2}}
$$

for $s_{i-1}<s<s_{i}$. Now by definition of $e_{2}\left(s_{i}\right)$ and $e_{2}(s)$ for $s_{i}<s<s_{i+1}$ for the case where $m=m\left(s_{i}\right)$ is odd, (4) holds at $s=s_{i}(h=0)$ and for $s_{i}<s<s_{i+1}$ $(h>0)$ as well. This shows that the $i$-th component of $e_{2}(s)$ is given by the right hand side of (4) which is of class $C^{\infty}$ in the neighborhood $U$ of $s_{i}$.

Next we consider the case where $m=m\left(s_{i}\right)$ is even. We shall assume that $e_{2}(s)$ is given by (3) for $s_{i-1}<s<s_{i}$. As $m$ is even this time, we get

$$
i \text {-th component of } e_{2}(s)=-\frac{a^{i}+h \eta^{i}(h)}{\left(\sum_{i=1}^{3}\left(a^{i}+h \eta^{i}(h)\right)^{2}\right)^{1 / 2}}
$$

for $s_{i-1}<s<s_{i}\left(h=s-s_{i}<0\right)$. By our rule of defining $e_{2}(s)$, (5) holds for $s_{i}<s<s_{i+1}(h>0)$ and at $s=s_{i}(h=0)$ as well. Thus the $i$-th component of $e_{2}(s)$ is given by the right hand side of (5) which is of class $C^{\infty}$ in the neighborhood $U$ of $s_{i}$. We have concluded the proof that $e_{2}(s)$ is of class $C^{\infty}$ on the whole interval $L$.

We finally show that the vector function $e_{2}(s)$ is uniquely determined up 
to a sign on the whole interval $L$; this will prove that the function $k(s)=\left(e_{1}^{\prime}(s), e_{2}(s)\right)$ is unique up to a sign and that the function $w(s)$ is uniquely determined by $C$ since $w(s)=-\left(e_{2}(s)\right.$, $\left.e_{3}^{\prime}(s)\right)$ does not change if we change $e_{2}(s)$ into $-e_{2}(s)$. Now assume that we have $\overline{e_{2}}(s), \overline{e_{3}}(s)=\overline{e_{1}}(s)$ $\times \bar{e}_{2}(s)$ such that Frenet equations

$$
\begin{aligned}
& \bar{e}_{1}^{\prime}=\overline{k e_{2}} \\
& \overline{e_{2}^{\prime}}=-\overline{k e_{1}}+\overline{w e_{3}} \\
& \overline{e_{3}^{\prime}}=-\bar{w} \overline{e_{2}}
\end{aligned}
$$

for suitably chosen functions $\bar{k}$ and $\bar{w}$. From the first equation we have $|\bar{k}(s)|=\left\|e_{1}^{\prime}(s)\right\|=|k(s)|$. On each subinterval $\left(s_{i}, s_{i+1}\right)$, neither $k(s)$ nor $k(s)$ has a zero point. According as $k(s)>0$ or $k(s)<0$ on $\left(s_{i}, s_{i+1}\right)$, we have $e_{2}(s)=e_{1}^{\prime}(s) /\left\|e_{1}^{\prime}(s)\right\|$ or $e_{2}(s)=-e_{1}^{\prime}(s) /\left\|e_{1}^{\prime}(s)\right\|$. The situation is similar for $e_{2}(s)$. Thus if $k(s)$ and $\bar{k}(s)$ have the same sign on $\left(s_{i}, s_{i+1}\right)$, we have $e_{2}(s)=$ $\overline{e_{2}}(s)$, otherwise, $e_{2}(s)=-\overline{e_{2}}(s)$. At $s=s_{i}$ we have either $e_{2}\left(s_{i}\right)=\overline{e_{2}}\left(s_{i}\right)$ or $e_{2}$ $\left(s_{i}\right)=-\overline{e_{2}}\left(s_{i}\right)$. From the continuity we have $e_{2}(s)=\overline{e_{2}}(s)$ on $L$ or $e_{2}(s)=-$ $\overline{e_{2}}(s)$ on $L$, concluding the whole proof of our theorem.

3. Remarks. We first give an example of a curve of class $C^{\infty}$ which is neither normal nor a Frenet curve. Let $f(t)$ ke a $C^{\infty}$-function defined by $f(t)=e^{-1 / t^{2}}$ for $t<0$ and $f(t)=0$ for $t \geqq 0$. Similarly, let $g(t)$ ke defined by $g(t)=0$ for $t \leqq 0$ and $g(t)=e^{-1 / t^{2}}$ for $t>0$. We define a curve $C$ by $x^{1}(t)=$ $f(t), x^{2}(t)=t, x^{3}(t)=g(t)$ for $-1 \quad t \quad 1$. $C$ is a regular curve of class $C^{\infty}$ but not normal (the point corresponding to $t=0$ is singular). It is easy to see that $C$ is not a Frenet curve (for $t<0$, the vector $e_{3}(s)$ is perpendicular to the $x^{1} x^{2}$-plane, while for $t>0$ it is perpendicular to the $x^{2} x^{3}$-plane so that it is impossible to define $e_{3}(s)$ at the point $=0$ to get a continuous vector family $e_{3}(s)$ on the whole $\left.L\right)$.

On the other hand, given arbitrary functions $k(s)$ and $w(s)$, both of class $C^{\infty}$ on an interval $L$, there exists a Frenet curve of class $C^{\infty}$ for which the Frenet equations are valid with given functions $k$ and $w$. This curve, unique within to a motion in the space, may not le normal. In fact, by taking a function $k(s)$ which vanishes together with all its derivatives at a certain point, we get a Frenet curve which is not normal.

Although a pair of functions $k(s)$ and $w(s)$ determine a Frenet curve $C$, we should be cautioned against defining $w(s)$ to be the torsion of $C$. If $k$ is constantly zero, the curve $C$ will be a line segment whatever function w may be, while it is geometrically natural to define the torsion of a line segment to be zero. The same remark is to be applied to any line segment which 
may be contained in the curve $C$. In this connection, the following remark is a consequence of our results. If the function $k(s)$ satisfies the condition that for each $s_{0} \in L$, there is an integer $m$ such that the $m$-th derivative $k^{(m)}\left(s_{0}\right)$ is not zero, then the Frenet curve determined by $k(s)$ and an arbitrary function $w(s)$ is normal and hence the Frenet family of moving frames is essentially unique by the theorem. This shows that the function $w(s)$, uniquely determined by the curve $C$ as a function which appear in the Frenet equations of $C$, may be defined to be the torsion of $C$ without any ambiguity.

We finally give a geometric meaning of the change of sign of $k(s)$ in Corollary 1. Let $C$ ke a normal curve of class $C^{\infty}$. If $k\left(s_{0}\right) \neq 0$ and $w(s)$ $\neq 0$, then the curve $C$ lies on different sides of the osculating planes in a neighborhood of the point $s=s_{0}$, as is known in the classical theory. When $k\left(s_{0}\right)=0$, the osculating plane is still defined to be the plane determined by $e_{1}\left(s_{0}\right)$ and $e_{2}\left(s_{0}\right)$. Now assume that $k\left(s_{0}\right)=0$ but $w\left(s_{0}\right) \neq 0$. If $m\left(s_{0}\right)$ is odd, the situation is the same as the case where $k\left(s_{0}\right) \neq 0$, while if $m\left(s_{0}\right)$ is even, the curve $C$ lies on one side of the osculating plane in a neighborhood of the point. This follows from the following argument. Let $m=m\left(s_{0}\right)$ and let us expand $x(s)=\left(x^{i}(s)\right)$ in the form

$$
\begin{aligned}
x(s)=x\left(s_{0}\right) & +\left(s-s_{0}\right) e_{1}\left(s_{0}\right)+\left(s-s_{0}\right)^{m+1} /(m+1) ! e_{1}^{(m)}\left(s_{0}\right) \\
& +\left(s-s_{0}\right)^{m+2} /(m+2) !\left\{e_{1}^{(m+1)}\left(s_{0}\right)+\varepsilon\right\},
\end{aligned}
$$

where $\varepsilon \rightarrow 0$ as $s \rightarrow s_{0}$.

We see that the inner product $\left(x(s)-x\left(s_{0}\right), e_{3}\left(s_{0}\right)\right)$ is equal to $\left(s-s_{0}\right)^{m_{+2}} /$ $(m+2) !\left\{\left(e_{1}^{(m+1)}\left(s_{0}\right), e_{3}\left(s_{0}\right)\right)+\varepsilon^{\prime}\right\}$. On the other hand, differentiating $\left(e_{1}^{\prime}, e_{3}\right)=0$ $m$ times, we get $m\left(e_{1}^{(m)}\left(s_{0}\right), e_{3}^{\prime}\left(s_{0}\right)\right)+\left(e_{1}^{(m+1)}\left(s_{0}\right), e_{3}\left(s_{0}\right)\right)=0$ so that $\left(e_{1}^{(m+1)}\left(s_{0}\right), e_{3}\left(s_{0}\right)\right)$ $=-m\left(e_{1}^{(m)}\left(s_{0}\right), e_{3}^{\prime}\left(s_{0}\right)\right)=m\left(e_{1}^{(m)}\left(s_{0}\right), w\left(s_{0}\right) e_{2}\left(s_{0}\right)\right) \neq 0$ since $e_{2}\left(s_{0}\right)$ is equal to the unit vector in the same direction as $e_{1}^{(m)}\left(s_{0}\right)$ and $w\left(s_{0}\right) \neq 0$ by assumption. Thus we have

$$
\left(x(s)-x\left(s_{0}\right), e_{3}\left(s_{0}\right)\right)=\left(s-s_{0}\right)^{m+2}\left(a+\varepsilon^{\prime \prime}\right)
$$

where $a \neq 0$ and $\varepsilon^{\prime \prime} \rightarrow 0$ as $s \rightarrow s_{0}$, from which our assertion is clear.

\section{NAGOYA UNIVERSITY.}

